\title{
Bilingual word recognition in a sentence context
}

\section{Eva Van Assche*, Wouter Duyck and Robert J. Hartsuiker}

Department of Experimental Psychology, Ghent University, Ghent, Belgium

\section{Edited by:}

Jon Andoni Dunabeitia, Basque Center on Cognition, Brain and Language, Spain

Reviewed by:

Clara D. Martin, Universitat Pompeu Fabra, Spain

Yan Jing Wu, Bangor University, UK

*Correspondence:

Eva Van Assche, Department of Experimental Psychology, Ghent University, Henri Dunantlaan 2, 9000

Ghent, Belgium.

e-mail: eva.vanassche@ugent.be
This article provides an overview of bilingualism research on visual word recognition in isolation and in sentence context. Many studies investigating the processing of words out-of-context have shown that lexical representations from both languages are activated when reading in one language (language-non-selective lexical access). A newly developed research line asks whether language-non-selective access generalizes to word recognition in sentence contexts, providing a language cue and/or semantic constraint information for upcoming words. Recent studies suggest that the language of the preceding words is insufficient to restrict lexical access to words of the target language, even when reading in the native language. Eye tracking studies revealing the time course of word activation further showed that semantic constraint does not restrict language-non-selective access at early reading stages, but there is evidence that it has a relatively late effect. The theoretical implications for theories of bilingual word recognition are discussed in light of the Bilingual Interactive Activation+ model (Dijkstra and van Heuven, 2002).

Keywords: bilingualism, visual word recognition, sentence processing, eye tracking

\section{INTRODUCTION}

The task of reading is omnipresent in everyday life. People can read in their native language without apparent difficulty. It takes a skilled reader only a few hundred milliseconds to recognize a word. This is extremely fast given that the mental lexicon contains tens of thousands of words from among which the correct word has to be identified. Furthermore, many people have knowledge of more than one language. Recently, the process of reading by bilinguals has increasingly attracted the attention of the scientific community. Research on bilingualism includes issues such as: Are the words of one language activated when reading in the other? Are there any differences in cross-lingual activation between words presented in isolation and words presented in sentence context? What is the time course of cross-lingual activation and what factors may modulate this activation process? The most intuitively appealing idea would probably be that bilinguals have two separate lexicons that can be accessed selectively so that each language functions independently of the other. After all, most bilinguals can speak and read in each language without too many intrusions or errors (Poulisse and Bongaerts, 1994). However, in the last decade, more and more researchers have come to realize that "the bilingual does not equal the sum of two monolinguals" (Grosjean, 1989). Bilinguals do not recognize words in exactly the same way as monolinguals. It became clear that the two languages interact with each other when bilinguals are processing words in one language (e.g., Dijkstra et al., 1999; van Hell and Dijkstra, 2002; Duyck, 2005; Van Assche et al., 2009).

In this review, we focus on visual word recognition research in bilinguals and the lexical organization of the bilingual language system. First, we briefly summarize the main experimental findings in isolated word recognition. Then, we present the recently developed research line on bilingual word recognition in sentence contexts. Next, we discuss the most influential theoretical accounts on the lexical organization of the bilingual language system and we present the theoretical implications of the research presented in this review for theories of bilingual word recognition, in particular the Bilingual Interactive Activation $+(\mathrm{BIA}+)$ model (Dijkstra and van Heuven, 2002). Finally, we discuss future work directions for the study of the bilingual language system.

\section{BILINGUAL VISUAL WORD RECOGNITION IN ISOLATION}

An important issue in bilingualism research concerns the question of whether reading a word activates lexical representations in both languages, or in only the contextually relevant (target) language. Most of the research on this issue has focused on the cross-lingual interactions between orthographic representations. Evidence has accumulated that representations from both languages are activated in parallel (e.g., van Heuven et al., 1998; Dijkstra et al., 1999; van Hell and Dijkstra, 2002; Duyck, 2005; Van Assche et al., 2009). To our knowledge, Caramazza and Brones (1979) were the first to find evidence for the currently dominant theory that lexical representations in both languages are activated when reading in one language (i.e., language-non-selective access). In this study, Spanish-English bilinguals performed a lexical decision task (in which participants decide whether a string of letters is a word or a non-word) in their second language (L2). They found that bilinguals responded more quickly to cognates (i.e., translation equivalents with full or partial form overlap, e.g., Spanish-English piano-piano, eco-echo) than to matched non-cognates. This cognate facilitation effect is commonly attributed to the fact that a L2 cognate word also activates the L1 lexical representation of the cognate, mapped onto the same semantic representation, to a certain degree (see Dijkstra and van Heuven, 2002; Dijkstra et al., 2010, for more information on the representational structure of cognates). The cross-lingual activation of these representations speeds up the recognition of cognates compared to non-cognates.

Later, several studies have replicated this cognate facilitation effect in L2 for words presented out-of-context (e.g., Dijkstra 
et al., 1999; Lemhöfer and Dijkstra, 2004; Duyck et al., 2007). In Lemhöfer et al. (2004), this effect is shown to even accumulate over languages. Lemhöfer et al. tested Dutch-English-German trilinguals performing a German (L3) lexical decision task and reported faster responses for L1-L2-L3 cognates than for L1-L3 cognates. Surprisingly, cognate facilitation even occurs when bilinguals perform a lexical decision task in their native and dominant language (L1; e.g., van Hell and Dijkstra, 2002; Van Assche et al., 2009). van Hell and Dijkstra (2002) investigated the influence of L2 and L3 on reading in the L1. Two groups of Dutch-English-French trilinguals with low and high proficiency in French performed a Dutch lexical decision task. The critical stimuli were L1-L2 cognates and L1-L3 cognates. For both groups of trilinguals, results yielded faster lexical decisions for L1-L2 cognates than for noncognates. However, only the trilinguals who were highly proficient in French showed cognate facilitation for L1-L3 cognates. These results provide strong evidence for language-non-selective access in the bilingual lexicon because the non-dominant languages exert an influence on the dominant L1. A minimal proficiency in the non-dominant language seems necessary however in order to obtain cross-lingual activation effects.

Other evidence for language-non-selective access comes from studies investigating the recognition of interlingual homographs (i.e., words that have the same orthographic form in both languages but that have a different meaning, e.g., Dutch-English room, meaning cream in Dutch; e.g., Dijkstra et al., 1998, 1999, 2000; Kerkhofs et al., 2006). In Dijkstra et al. (2000), Dutch-English bilinguals performed a go/no-go task in which they had to press a button only if the presented word was an English word. Reaction times for interlingual homographs were slower than for control words. Apparently, the Dutch reading of the homograph was activated and interfered with the recognition of the English word. The size and direction of this interlingual homograph effect can be modulated by task requirements, language intermixing and relative frequency of the homograph in the two languages. For instance, Dijkstra et al. (1998) observed facilitation for interlingual homographs when Dutch-English bilinguals performed a generalized lexical decision task (giving a yes-response when a word of either language was presented). It seems that participants responded as soon as one reading of the homograph was available, or even on the basis of the summed activity in the bilingual language system generated by the two readings of the homograph.

In addition to these cognate and homograph studies, there is further evidence for cross-lingual activation of lexical representations from neighborhood studies (e.g., van Heuven et al., 1998) and masked priming studies (e.g., Bijeljac-Babic et al., 1997). Monolingual studies have shown that word processing is influenced by the number (density) of orthographic neighbors (i.e., words differing by a single letter from the target, Coltheart et al., 1977; e.g., house is an intralingual neighbor of mouse) and their frequency (e.g., Grainger et al., 1989; Segui and Grainger, 1990). van Heuven et al. (1998) examined the claim of an integrated lexicon and language-non-selective lexical access by investigating whether word neighbors in both languages [e.g., book is a cross-lingual neighbor of the Dutch word rook (smoke)] affect word recognition. The results from Dutch-English bilinguals' performance on two progressive demasking tasks showed that a higher number of Dutch word neighbors resulted in slower responses to English target words. This inhibitory effect of the number of neighbors was also present for word identification in the L1: Dutch-English bilinguals needed more time to identify a Dutch word with many English neighbors than a Dutch word with few English neighbors. van Heuven et al. also tested whether these results generalized to different task situations. As in the progressive demasking experiments, results of a generalized lexical decision task showed significant inhibition from Dutch neighbors on English word recognition. However, there was no effect of English neighbors on Dutch words. This suggests that the strength of neighborhood density effects is task dependent. An English lexical decision task with Dutch-English bilinguals showed an inhibitory effect from Dutch neighborhood on lexical decision times. This factor did not influence the responses of English monolinguals, ensuring that this effect was not due to any uncontrolled stimulus characteristics.

Other evidence for neighborhood density effects between languages comes from Bijeljac-Babic et al. (1997). They used the masked priming paradigm to test whether the inhibitory priming effect of orthographic neighbors on visual word recognition in monolinguals (e.g., Segui and Grainger, 1990) generalized to bilinguals. In Experiment 1, highly proficient French-English bilinguals made lexical decisions to L2 target words or non-words preceded by words from the same or a different language. Within each prime language condition, target words were preceded by either orthographically related primes (e.g., less-LOSS; joie-JOIN) or unrelated primes (sore-LOSS; acte-JOIN). When prime and target were from the same language, lexical decisions were slower after related primes than unrelated primes. More importantly, the same inhibition effect was found when prime and target were from different languages, providing evidence for language-non-selective access to the bilingual lexicon. In Experiment 2, the target language was changed and a different set of prime-target stimuli was tested in balanced and unbalanced bilinguals and in French monolinguals. The within-language effect was present in all three groups, while the between-language effect was larger for the balanced than for the unbalanced bilinguals. The French monolinguals showed no effect of English word primes. These cross-lingual activation effects from (masked) neighborhood studies strongly support the hypothesis of language-non-selective access to an integrated lexicon, even when subjects are performing a monolingual task. Note that converging evidence for language-non-selective access has also been obtained in other domains such as auditory word recognition (e.g., Spivey and Marian, 1999; Weber and Cutler, 2004; Lagrou et al., 2011) and word production (e.g., Costa et al., 1999).

We can conclude that there is a now a consensus in the bilingual literature about language-non-selective access of words in the two languages. However, in all of the studies discussed above, word recognition was always investigated for words presented out-of-context, using lab tasks (e.g., lexical decision) as operationalizations of reading. One of the key research questions for future bilingualism studies is whether these findings on lexical interactions between languages also generalize to word recognition in sentence contexts. The next section discusses the pioneer studies that have recently begun to assess this issue. 


\section{BILINGUAL VISUAL WORD RECOGNITION IN SENTENCES}

Whereas most studies on lexical autonomy have investigated the recognition of isolated words, word recognition rarely occurs outof-context. People usually read words embedded in meaningful sentences (e.g., in a newspaper article). The ecological validity of the studies on isolated word recognition can be put to the test by examining word recognition in sentences. The processing of words in isolation may differ in important ways from word processing during sentence reading. For instance, it is possible that the presentation of words in a sentence context restricts lexical activation to words of the target language only. This would actually be quite an efficient strategy to speed up word recognition, because it reduces the number of lexical candidates. And, indeed, in the monolingual domain, it has been shown that semantic and syntactic restrictions imposed by a sentence are used to speed up recognition of upcoming words (e.g., Schwanenflugel and LaCount, 1988). For instance, many studies have shown that context modulates lexical access for ambiguous words (e.g., bank as a riverside or a financial institution; e.g., Binder and Rayner, 1998). Also, previous research has shown that words embedded in a semantically constraining sentence context are processed faster than words embedded in a neutral sentence context (e.g., Stanovich and West, 1983; Rayner and Well, 1996). These monolingual studies indicate that sentence context can restrict semantic, syntactic, and lexical activation for word appearing later in the sentence.

The question now is whether such sentence context effects in monolinguals are also used by bilinguals to speed up lexical search through representations belonging to two different languages. Although there is one early study of Altarriba et al. (1996) that investigated word recognition in a sentence context for mixedlanguage sentences, all other studies examining bilingual sentence reading were carried out only very recently (e.g., Elston-Güttler et al., 2005; Schwartz and Kroll, 2006; Duyck et al., 2007; van Hell and de Groot, 2008; Libben and Titone, 2009; Van Assche et al., 2009, 2011; Titone et al., 2011).

\section{L2 PROCESSING}

In these studies investigating bilingual sentence reading, the cognate or interlingual homograph effect has often been used as a marker of non-selective activation. In a semantic priming study, Elston-Güttler et al. (2005) showed that cross-lingual activation is very sensitive to the influence of a sentence context and the previous activation state of the two languages. German-English bilinguals were presented with relatively low-constraint sentences in which a homograph (e.g., The woman gave her friend a pretty GIFT; gift meaning poison in German) or a control word was presented at the end (e.g., The woman gave her friend a pretty SHELL). The sentence was then replaced by a target word for lexical decision (poison). Targets were recognized faster after the related homograph sentence than after the unrelated control sentence, but only in the first block of the experiment and only for participants who saw a German film prior to the experiment, boosting L1 activation. This suggests that the L1 meaning of the homograph was activated while reading L2 sentences, but only after boosting L1 activation and for a limited amount of time because, as Elston-Güttler et al. put it, the bilingual language system quickly "zooms into" the L2 processing situation.
Furthermore, recordings of event-related potentials (ERPs), time-locked to the target word, showed this semantic priming effect in the modulations of the N200 and N400 components. The N200 component in the 150- to 250-ms time window has been linked to word access and/or orthographic processing (e.g., Bentin et al., 1999; but see Connolly and Phillips, 1994, where the N200 has also been linked to phonological processing). Elston-Güttler et al. (2005) suggested a translational word form link between giftpoison so that lexical access of the target poison is faster after the prime gift. The N400 component, present in the time window from 300 to $500 \mathrm{~ms}$, has been linked to semantic integration processes (e.g., Brown and Hagoort, 1993). Target words (poison) are easier to integrate and therefore less negative in the N400 amplitude after a related prime (the L1 meaning of the homograph gift) than after an unrelated one (shell). This study showed that sentence context can prevent the activation of the homograph's non-target language representation and that this effect is very sensitive to task circumstances.

The study of Schwartz and Kroll (2006) tested cognate and homograph effects in Spanish-English bilinguals. They presented target words in low- and high-constraint sentences to investigate how the mere presentation of words in a sentence context, and the semantic constraint it provides, modulates language-nonselective activation in the bilingual lexicon. The words of the sentence were presented using rapid serial visual presentation and the target word (printed in red) had to be named. No homograph effects were found in either low- or high-constraint sentences, but less proficient bilinguals made more naming errors, especially in low-constraint sentences. These results for homographs were somewhat inconclusive and in this respect, it should be noted that results for interlingual homograph effects in isolation (e.g., Dijkstra et al., 2000) were also not always consistent and seem very sensitive to specific characteristics of the task. Therefore, cognate facilitation may be a more reliable marker of cross-lingual activation. Schwartz and Kroll observed cognate facilitation in low-constraint sentences, but not in high-constraint ones. This suggests that the semantic constraint of a sentence may restrict cross-lingual activation effects.

Similar results on cognate effects were obtained by van Hell and de Groot (2008) for Dutch-English bilinguals in an L2 lexical decision task and a translation task in forward (from L1 to L2) or in backward direction (from L2 to L1). Cognate facilitation was shown after the presentation of a low-constraint sentence, but cognate effects were no longer observed in high-constraint sentences in the lexical decision task and strongly diminished in the translation tasks.

In sum, data from studies using lexical decision, naming, or translation tasks suggest that the semantic constraint of a sentence modulates bilingual lexical access, reducing, or nullifying crosslingual activation effects. However, this is possibly the result of processes occurring after lexical access had taken place. Lexical decision tasks may involve decision-making strategies or postlexical checking strategies. In the same way, naming requires a production component. As a result, these processes might disguise the actual effects reflecting lexical access in bilinguals. It is therefore important to explore this issue using more sensitive measurements such as eye tracking. This method has several important 
advantages over lexical decision or naming. First, it allows reading as in everyday life and thereby provides the most natural experimental operationalization of reading. Second, there is no need for any overt response (e.g., as in lexical decision) that may be subject to strategic factors not directly related to word recognition. And finally, it allows to investigate the time course of lexical activation by dissociating several early (reflecting initial lexical access) and late reading time measures (reflecting higher-order processes; Rayner, 1998). Early measures typically include first fixations (i.e., the duration of the first fixation on the target word) and gaze durations (i.e., the sum of fixations from the moment the eyes land on the target for the first time until they move off again). Late reading time measures such as go-past times (i.e., the time elapsing from encountering a given target for the first time until a region to the right of the target is fixated) also include regressions originating from the target word.

The study of Duyck et al. (2007) used the eye tracking methodology to investigate the time course of cross-lingual activation effects in L2 sentence reading. Duyck et al. tested Dutch-English bilinguals while they read low-constraint sentences in which the cognate or its control were embedded (e.g., Hilda bought a new RING-COAT and showed it to everyone; ring is a cognate; coat is a control word). A pretest ensured that there were no differences in predictability between the cognate and control conditions. There was cognate facilitation from 249 ms onward after first encountering the target on early and late reading time measures, but only for identical cognates (i.e., cognates with identical orthographies across languages, e.g., ring-ring) and not for non-identical ones (e.g., schip-ship). The results indicate that when cross-lingual overlap was not complete, the cognate effect was not strong enough to be visible in a sentence. This shows that the amount of crosslingual activation is a function of the similarity between the translation equivalents. Furthermore, the eye movement results indicate that the cross-lingual activations in the bilingual lexicon responsible for the cognate effect occur early in word recognition because cognate facilitation was already present on the first fixation of the target, and remained present in later eye tracking measures.

Van Assche et al. (2011) fine-tuned the distinction between identical and non-identical cognates of Duyck et al. (2007) by calculating the degree of orthographic overlap on van Orden's (1987) word similarity measure for each cognate and control word on a scale from 0 to 1 (e.g., the English-Dutch identical cognate ring-ring: 1.00; non-identical cognate shoulder-schouder: 0.81; control witch-heks: 0.06). Targets were presented in lowand high-constraint sentences. A cloze probability test ensured that cognates and controls were equally predictable in the sentences. In low-constraint sentences, discrete cognate facilitation (cognate vs. control) was again observed on first fixation durations, gaze durations and go-past times. Interestingly, this was shown to be a gradual and continuous effect: reading times were faster as the cross-lingual orthographic overlap between translation equivalents increased. In addition, cognate facilitation was already present on skipping rates (i.e., the probability that the word was not fixated): cognates were skipped more often than noncognates, arguably reflecting the early origin of these cross-lingual activation effects in the time course of word processing. More importantly, Van Assche et al. also examined how a strong semantic context modulates lexical activation spreading between languages in the bilingual lexicon by presenting cognates in high-constraint sentences. Cognate effects were observed in high-constraint sentences on both early and late measures and were present both when cognate status was taken as a discrete dichotomous variable and as a continuous variable. A control experiment with English monolinguals in which cognate effects disappeared ensured that the effects were genuinely due to the Dutch-English cross-lingual overlap. Thus, this study clearly finds evidence for cross-lingual interaction effects in the presence of a semantically constraining sentence at any stage of word recognition. This contrasts with the results of previous studies on this topic (e.g., Schwartz and Kroll, 2006; van Hell and de Groot, 2008). It seems that the use of the time-sensitive eye tracking measures uncovers the early interaction effects that were not observed in the naming task of Schwartz and Kroll (2006) or the lexical decision and translation tasks of van Hell and de Groot (2008).

The absence of an interaction between semantic constraint effects and the time course of cross-lingual lexical interactions (Van Assche et al., 2011) contrasts with the eye movement results of Libben and Titone (2009) who found cognate facilitation in semantically constraining sentences only on early comprehension measures. French-English bilinguals were presented with formidentical cognates and homographs in English sentences of low and high semantic constraint. Results showed cognate facilitation and homograph interference on all early and late measures in low-constraint sentences. However, in high-constraint sentences, these cross-lingual interaction effects were only observed on early stage reading time measures (i.e., first fixations, gaze durations, and skipping rates for cognates; gaze durations for homographs), but no effects were obtained on late stage measures. Libben and Titone suggested that lexical access in bilinguals is non-selective at early word processing stages, but that this dual-language activation is rapidly resolved by top-down factors (e.g., semantics) at later stages of comprehension.

Several factors may explain the inconsistent results across these studies. It is not the case that Van Assche et al. (2011) used a weaker semantic constraint manipulation. On the contrary, cloze probabilities in Van Assche et al. ( 0.86 for cognates and 0.89 for controls) were stronger than these in Libben and Titone (2009; 0.48 for cognates and 0.49 for controls). The specific bilingual population may be a key factor responsible for the different results. The bilinguals tested by Van Assche et al. were less balanced in their percentage of daily use of L1 and L2 and had acquired their L2 English later than Libben and Titone's. Therefore, Titone et al. (2011) argued that the L1 of the participants in Van Assche et al. may be more strongly activated, leading to greater L1-to-L2 cross-language activation so that semantic context may be insufficient to diminish cross-language activation.

In conclusion, these studies on L2 sentence processing indicate that the mere presentation of words in a sentence context and the language cue it provides does not nullify dual-language activation in the bilingual language system. Mixed results have been obtained for semantically constraining sentences, but recent studies using time-sensitive eye movement recordings suggest that even a strong semantic context does not necessarily eliminate 
cross-lingual activation effects, at least for early interaction effects reflected in early reading time measures.

\section{L1 PROCESSING}

Although the vast majority of studies on bilingual word recognition have focused on L2 processing, there are a few studies that have investigated cross-language activation during nativelanguage reading (e.g., Van Assche et al., 2009; Titone et al., 2011). van Hell and Dijkstra (2002) were the first to show that cognate facilitation for words out-of-context can be obtained in an exclusively native-language context. Van Assche et al. (2009) replicated this cognate effect in L1 for words out-of-context and they also investigated how a linguistic context provided by a sentence may restrict this cross-lingual activation. Dutch-English bilinguals were presented with low-constraint sentences that could include both the cognate and its control [e.g., Ben heeft een oude OVEN/LADE gevonden tussen de rommel op zolder (Ben found an old OVEN/DRAWER among the rubbish in the attic); oven is a Dutch-English cognate; lade is a control word]. Cognate facilitation was observed on early reading time measures, both as a discrete effect of cognates vs. controls and as a continuous facilitation effect of cross-lingual orthographic overlap. This implies that even when native-language processing is concerned, bilinguals are different from monolinguals: the mere knowledge of a second language affects a highly automated skill as sentence reading in the mother tongue. These findings provide strong evidence for language-non-selective access in the bilingual lexicon.

Titone et al. (2011) tested whether semantic constraint would modulate cross-language activation during L1 reading. Formidentical cognate facilitation and interlingual homograph interference was used as a marker of cross-lingual interactions. In a first experiment, English-French bilinguals read low- and highconstraint L1 sentences (e.g., Because of the bitter custody battle over the kids, the expensive DIVORCE was a disaster; divorce is an English-French cognate) while eye movements were recorded. Cognate facilitation was present on early reading time measures. This effect was independent of contextual constraint, but it was modulated by $\mathrm{L} 2$ age of acquisition: only bilinguals who acquired their L2 early in life showed cognate facilitation. The L2 age of acquisition did not affect the size of cognate facilitation on late reading time measures, but here, semantic constraint did: cognate effects were smaller in high- than low-constraint sentences.

In Experiment 2, Titone et al. (2011) intermixed French L2 sentences with the experimental English L1 sentences to assess whether making L2 more salient would increase cognate facilitation and interlingual homograph interference during $\mathrm{L} 1$ reading. And indeed, cognate effects on late reading time measures did not diminish in high-constraint sentences when L1 and L2 sentences were intermixed. Titone et al. suggested that the inclusion of the L2 sentences may have increased cross-language activation during $\mathrm{L} 1$ reading, which may have countered the effect of semantic constraint.

The homograph results showed no interference effects for first fixations, gaze durations, and go-past times in Experiments 1 and 2. There was, however, homograph interference for total reading times. It is striking how this pattern of results differs from the cognate results and the homograph results in an earlier study of L2 reading (Libben and Titone, 2009) because cognate and homograph effects are assumed to originate both from cross-lingual activation patterns in the bilingual language system. A possible explanation proposed by Titone et al. (2011) is that homographs and cognates are represented differently at the lexical level.

Summarizing, Van Assche et al. (2009) showed that a nondominant language may affect native-language sentence reading, both at the earliest and at later reading stages. Titone et al. (2011) observed this cross-language activation at early reading stages only when the L2 was acquired early in life. They also showed that the semantic constraint provided by a sentence can attenuate cross-language activation at later reading stages.

\section{THEORETICAL ACCOUNTS ON LEXICAL ORGANIZATION IN BILINGUALS}

A theoretical explanation of the cross-lingual activation effects discussed in this review can be framed within bilingual language processing models such as the BIA+ model (Dijkstra and van Heuven, 2002). It is the successor of the original BIA model (Dijkstra and van Heuven, 1998), which was a bilingual extension of the Interactive Activation model (McClelland and Rumelhart, 1981). Two basic assumptions of the BIA+ model are that L1 and L2 words are represented in an integrated lexicon and that word recognition proceeds in a language-non-selective way. Upon the presentation of a word, orthographic, phonological, and semantic representations become activated (bottom-up) in both languages depending on the overlap with the input word. For homographs, orthographic representations in both languages will become strongly activated because of the identical orthography across languages, thereby activating two different semantic representations. Nonhomographic control words on the other hand, will only activate lexical representations in the target language. This difference in activation level for homographs and control words gives rise to the homograph effect. For cognate words on the other hand, it is the high degree of cross-lingual orthographic, phonological, and semantic overlap that results in the cognate effect. The crosslingual activation from these three codes speeds up the recognition of cognates compared to non-cognates.

Other theoretical accounts of the cognate effect attribute its origin to a morphological (e.g., Kirsner et al., 1993; SánchezCasas and García-Albea, 2005) or to a conceptual level (e.g., de Groot and Nas, 1991; van Hell and de Groot, 1998). For instance, Sánchez-Casas and García-Albea (2005) proposed that cognate translations share a morphological representation in bilingual memory whereas non-cognate translations have separate morphological representations in bilingual memory. Another account assumes that the conceptual representations of cognate translations are linked or shared across languages (e.g., van Hell and de Groot, 1998). The continuous effect of cognate status based on the degree of cross-lingual overlap in the two languages is more in line with the account that assumes cognate effects to arise from the convergent activation of orthographic, phonological, and semantic representations (e.g., van Hell and de Groot, 1998; Dijkstra and van Heuven, 2002), although a study of Lehtonen et al. (2006) also suggest a possibly different morphological representation for bilinguals and monolinguals. 
In the BIA+ model (Dijkstra and van Heuven, 2002), there is a representational layer containing two language nodes, one for each language. These language nodes function as language tags, indicating to which language an item belongs, and they also reflect the global lexical activity of each language. In the earlier BIA model (Dijkstra and van Heuven, 1998), language nodes also served other functions such as language filters dependent on experimental variables or collectors of contextual activation coming from outside the lexicon. The language nodes could then facilitate activation of target language words through the inhibition of non-target language words. In this way, language nodes could account for top-down effects to the word level, although simulations have shown that language nodes cannot inhibit non-target language words sufficiently to obtain language selective access from the beginning of word recognition. Later, it became clear that combining both representational and functional aspects of language processing in one mechanism was not tenable and language nodes' function became purely representational. With respect to sentence context effects, Dijkstra and van Heuven (2002) suggested that language nodes can be pre-activated by the sentence, but as language nodes cannot inhibit non-target language words sufficiently, the mere presentation of words in a sentence does not constrain language-non-selective activation.

In order to account for differences between experiments and non-linguistic context effects (e.g., task features, instructions, participant's expectations), a distinction is made between the word identification system (containing orthographic, phonological, and semantic representation) and the task/decision system. Linguistic context, arising from lexical, syntactic, or semantic restrictions (e.g., a sentence context) is assumed to directly affect the word identification system. Non-linguistic context on the other hand, is assumed to affect the task/decision system. Dijkstra and van Heuven (2002) present the word identification system as part of a larger system in which sentence parsing and language production are also represented (e.g., Levelt et al., 1999). As the sentence parsing system may directly interact with the word identification system, syntactic and semantic context information may affect word recognition. Indeed, they explicitly state that such linguistic context information may restrict language-non-selective activation in bilinguals. However, they do not specify the exact mechanism that can give rise to these predicted top-down effects.

\section{SUMMARY AND THEORETICAL IMPLICATIONS}

The studies on bilingual sentence processing reviewed in the present paper showed that markers of language-non-selective access (such as cognate facilitation) were not nullified in the presence of a sentence context. It thus seems that the language of the preceding words is an insufficient cue to restrict lexical access to words of the target language (e.g., Schwartz and Kroll, 2006; Duyck et al., 2007; van Hell and de Groot, 2008), even when reading in the mother tongue (e.g., Van Assche et al., 2009). Furthermore, eye tracking studies revealing the time course of activation showed that semantic constraint does not necessarily restrict nonselective activation (Van Assche et al., 2011), although there is evidence that it has a relatively late effect (e.g., Libben and Titone, 2009; Titone et al., 2011), and that it affects cross-lingual activation in lexical decision, naming, and translation studies (e.g.,
Schwartz and Kroll, 2006; van Hell and de Groot, 2008). The difference in result patterns across studies suggests that the interaction between lexical activation and sentence processing is dependent on several experimental factors such as task demands (e.g., lexical decision vs. eye tracking; Duyck et al., 2007; van Hell and de Groot, 2008), type of bilinguals tested, lexical characteristics (e.g., identical vs. non-identical cognates; Duyck et al., 2007), and stimulus list composition (e.g., Titone et al., 2011).

These findings have important implications for the further development of models of bilingual word recognition. The BIA+ model (Dijkstra and van Heuven, 2002) for example, does not specify how linguistic context may exert effects in the bilingual language system. They did suggest that the language of the preceding words in the sentence does not restrict lexical activation. Indeed, the pre-activation of the language nodes by a sentence is not sufficient to restrict lexical access because language nodes cannot inhibit words to a considerable extent. Instead, lexical activation depends on the similarity of the input word with the representations in the lexicon and on the resting-level activation of the representations. The fact that cross-lingual activation was preserved in low-constraint sentences in L2 (e.g., Schwartz and Kroll, 2006; van Hell and de Groot, 2008; Libben and Titone, 2009; Van Assche et al., 2011) and in L1 (Van Assche et al., 2009; Titone et al., 2011) provides strong support for the assumption of limited influence of the sentence's language.

Furthermore, Dijkstra and van Heuven (2002) argued that the word identification system interacts with higher levels of linguistic processing (such as parsing), but they did not specify an exact mechanism for these top-down interactions from semantics to the orthographic and phonological levels. Given the data discussed in this review, how may these top-down interactions be interpreted within the BIA + model? The reduction of homograph interference in high-constraint sentences (e.g., Libben and Titone, 2009) can easily be accounted for in the BIA+ model because it predicts that the semantic level feeds back activation to the orthographic level. As homographs have distinct semantic representations in each language, the semantic representation activated by the sentence context feeds back to the orthographic level so that the competition between the identical orthographic representations of homographs is resolved faster.

In order to explain the reduced cognate effects in semantically constraining sentences (e.g., Schwartz and Kroll, 2006; van Hell and de Groot, 2008), additional assumptions are needed regarding the role of semantic constraint on lexical activation. For instance, monolingual studies indicate that sentence context can restrict semantic, syntactic, and lexical activation for words appearing later in the sentence (e.g., Stanovich and West, 1983; Schwanenflugel and LaCount, 1988). Extrapolating this to bilinguals, we propose that, similar to the view of Altarriba et al. (1996), a semantically constraining sentence not only generates semantic and syntactic restrictions for upcoming words, but that these restrictions also result in the pre-activation of lexical representations. This may speed up lexical access for cognates so much that the convergent bottom-up activation from non-target lexical representations no longer exerts an effect.

Furthermore, recent eye tracking studies testing cognates (e.g., Libben and Titone, 2009; Van Assche et al., 2009; Titone et al., 2011) 
showed clear cognate effects in early reading stages (reflected in measures such as first fixation duration and gaze duration), indicating that lexical restrictions only exert an influence during later stages of word recognition and after initial language-non-selective access had taken place. At present, it is not clear how the BIA+ model can explain the lexical restrictions generated by the sentence. The function of the language node may have an important role in this issue, but language nodes in the BIA + model only have a representational function and cannot substantially inhibit words in the non-target language. In order to account for the lexical restrictions, it may be necessary to assume a feedback mechanism from the language nodes to the orthographic level, so that language nodes can have a direct effect on lexical selection. This way, we assure the possibility of selectivity, constrained by semantic and lexical restrictions provided by a sentence context, in the fundamentally language-non-selective bilingual language system.

It seems that the top-down modulation from semantics to the orthographic level only occurs during later stages of word recognition, but this conclusion is not fully supported by the empirical evidence. First, Van Assche et al. (2011) obtained no such modulation of the cognate effect on late reading time measures (e.g., go-past time), suggesting a very limited role of these top-down restrictions. A possible, tentative explanation for the fact that Van Assche et al. observed cognate facilitation on late reading time measures may be that if readers do not make many regressions from the target word, early reading time measures will be similar because they are completely included in late measures. Indeed, early and late reading time measures differed much more in the eye tracking studies of Libben and Titone (2009) than in Van Assche et al. Second, Titone et al. (2011) showed reduced cognate facilitation on late reading time measures in Experiment 1, but not in Experiment 2 when non-target language filler sentence were included. This indicates that the inclusion of fillers increased cross-lingual activation and may have countered the effect of sentential constraint. Here, global language processing context may have influenced bilingual word recognition, just as in Elston-Güttler et al. (2005), and this may also be linked to the language mode theory (Grosjean, 1997): lexical access may be more or less selective depending on the language context and/or the bilinguals' expectations.

\section{FUTURE WORK DIRECTIONS}

For the further development of the BIA + model (Dijkstra and van Heuven, 2002) and other bilingual models, it is important

\section{REFERENCES}

Altarriba, J., Kroll, J. F., Sholl, A., and Rayner, K. (1996). The influence of lexical and conceptual constraints on reading mixed-language sentences: evidence from eye fixations and naming times. Mem. Cognit. 24, 477-492.

Bentin, S., Mouchetant-Rostaing, Y., Giard, M. H., Echalier, J. F., and Pernier, J. (1999). ERP manifestations of processing printed words at different psycholinguistic levels:

to note that the interactions between linguistic context and lexical variables in the BIA+ model (Dijkstra and van Heuven, 2002) may also interact with experimental/task factors (e.g., Duyck et al., 2007; van Hell and de Groot, 2008) or with participant characteristics such as age of acquisition of the L2 (e.g., Titone et al., 2011). For instance, it is important to examine whether the results generalize to other bilingual populations. For example, the bilinguals tested in Libben and Titone (2009) were more balanced and acquired their L2 earlier in life than Van Assche et al.'s (2011) bilinguals. A systematic test of the effects of proficiency and age of acquisition in future studies may help to explain whether these were the determining factors for the differences in results between these studies. Related to proficiency issues, it should be noted that many studies used self-ratings on reading, writing, speaking, and/or general proficiency. Although self-ratings provide an important indication of the proficiency level, in future studies, it is advisable to also use more direct measures to determine the L2 proficiency level such as measuring reaction times to words in both languages in lexical decision or naming tasks.

Future studies will also have to investigate how task effects influence the degree of language-non-selective access that is observed. There are important differences between results obtained with paradigms such as lexical decision, naming, and translation (e.g., Schwartz and Kroll, 2006; van Hell and de Groot, 2008) and those obtained with eye tracking (e.g., Libben and Titone, 2009; Van Assche et al., 2011). Only studies using eye tracking found evidence for cognate facilitation in semantically constraining sentences. It may well be that eye tracking constitutes a more sensitive paradigm. To examine this claim, Van Assche et al. (2011) ran an additional experiment in which the stimulus materials used in their eye tracking experiment were tested using the lexical decision paradigm of van Hell and de Groot (2008). They obtained cognate effects in low- and high-constraint sentences, but the latter effect was not very robust: cognate facilitation was weak and only emerged after testing many more bilinguals than van Hell and de Groot (2008) did. Another possibility, given in Libben and Titone (2009), is that lexical decision, naming, and/or translation tasks reflect comprehension processes subsequent to lexical access (during which cross-language activation is restricted by the semantic constraint for the target). Especially eye tracking may be sensitive enough to detect the earliest stages of word recognition and further studies are needed to clarify this issue.

Brown, C. M., and Hagoort, P. (1993). The processing nature of the N400: evidence from masked priming. $J$. Cogn. Neurosci. 5, 34-44.

Caramazza, A., and Brones, I. (1979). Lexical access in bilinguals. Bull. Psychon. Soc. 13, 212-214.

Coltheart, M., Davelaar, A., Jonasson, J. T., and Besner, D. (1977). "Access to the internal lexicon," in Attention and Performance, Vol. 6, ed. S. Dornic (New York: Academic Press), 535-555.
Connolly, J. F., and Phillips, N. A. (1994). Event-related potential components reflect phonological and semantic processing of the terminal word of spoken sentences. J. Cogn. Neurosci. 6, 256-266.

Costa, A., Miozzo, M., and Caramazza, A. (1999). Lexical selection in bilinguals: do words in the bilingual's two lexicons compete for selection? J. Mem. Lang. 41, 365-397. 
de Groot, A. M. B., and Nas, G. L. J. (1991). Lexical representation of cognates and noncognates in compound bilinguals. J. Mem. Lang. 30, $90-123$.

Dijkstra, T., Grainger, J., and van Heuven, W. J. B. (1999). Recognition of cognates and interlingual homographs: the neglected role of phonology. J. Mem. Lang. 41, 496-518.

Dijkstra, T., Miwa, K., Brummelhuis, B., Sappelli, M., and Baayen, H. (2010). How cross-language similarity and task demands affect cognate recognition. J. Mem. Lang. 62, 284-301.

Dijkstra, T., Timmermans, M., and Schriefers, H. (2000). On being blinded by your other language: effects of task demands on interlingual homograph recognition. $J$. Mem. Lang. 42, 445-464.

Dijkstra, T., and van Heuven, W. J. B. (1998). "The BIA model and bilingual word recognition," in Localist Connectionist Approaches to Human Cognition, eds J. Grainger and A. Jacobs (Mahwah, NJ: Lawrence Erlbaum Associates), 189-225.

Dijkstra, T., and van Heuven, W. J. B. (2002). The architecture of the bilingual word recognition system: from identification to decision. Biling. (Camb. Engl.) 5, 175-197.

Dijkstra, T., Van Jaarsveld, H., and Ten Brinke, S. (1998). Interlingual homograph recognition: effects of task demands and language intermixing. Biling. (Camb. Engl.) 1, 51-66.

Duyck, W. (2005). Translation and associative priming with cross-lingual pseudohomophones: evidence for nonselective phonological activation in bilinguals. J. Exp. Psychol. Learn. Mem. Cogn. 31, 1340-1359.

Duyck, W., Van Assche, E., Drieghe, D., and Hartsuiker, R. J. (2007). Visual word recognition by bilinguals in a sentence context: evidence for nonselective lexical access. J. Exp. Psychol. Learn. Mem. Cogn. 33, 663-679.

Elston-Güttler, K. E., Gunter, T. C., and Kotz, S. A. (2005). Zooming into L2: global language context and adjustment affect processing of interlingual homographs in sentences. Cogn. Brain Res. 25, 57-70.

Grainger, J., O’Regan, J. K., Jacobs, A. M., and Segui, J. (1989). On the role of competing word units in visual word recognition: the neighborhood frequency effect. Percept. Psychophys. $45,189-195$.

Grosjean, F. (1989). Neurolinguists, beware - the bilingual is not 2 monolinguals in one person. Brain Lang. 36, 3-15.

Grosjean, F. (1997). "Processing mixed language: issues, findings, and models," in Tutorials in Bilingualism: Psycholinguistic Perspectives, eds A. M. B. de Groot and J. F. Kroll (Mahwah, NJ: Erlbaum), 225-254.

Kerkhofs, R., Dijkstra, T., Chwilla, D. J., and de Bruijn, E. R. A. (2006) Testing a model for bilingual semantic priming with interlingual homographs: RT and N400 effects. Brain Res. 1068, 170-183.

Kirsner, K., Lalor, E., and Hird, K. (1993). "Exercise, meaning and morphology," in The Bilingual Lexicon, eds R. Schreuder and B. Weltens (Amsterdam: Benjamins), 215-248.

Lagrou, E., Hartsuiker, R. J., and Duyck, W. (2011). Knowledge of a second language influences auditory word recognition in the native language. $J$. Exp. Psychol. Learn. Mem. Cogn. 37, 952-965.

Lehtonen, M., Niska, H., Wande, E., Niemi, J., and Laine, M. (2006). Recognition of inflected words in a morphologically limited language: frequency effects in monolinguals and bilinguals. J. Psycholinguist. Res. $35,121-146$.

Lemhöfer, K., and Dijkstra, T. (2004). Recognizing cognates and interlingual homographs: effects of code similarity in language-specific and generalized lexical decision. Mem. Cogn. 32, 533-550.

Lemhöfer, K., Dijkstra, T., and Michel, M. C. (2004). Three languages, one echo: cognate effects in trilingual word recognition. Lang. Cogn. Process. 19, 585-611.

Levelt, W. J. M., Roelofs, A., and Meyer, A. S. (1999). A theory of lexical access in speech production. Behav. Brain Sci. 21, 1-38.

Libben, M. R., and Titone, D. A. (2009). Bilingual lexical access in context: evidence from eye movements during reading. J. Exp. Psychol. Learn. Mem. Cogn. 35, 381-390.

McClelland, J. L., and Rumelhart, D. E. (1981). An interactive activation model of context effects in letter perception. I. An account of basic findings. Psychol. Rev. 88, 375-407.
Poulisse, N., and Bongaerts, T. (1994). 1st Language use in 2nd-language production. Appl. Linguist. 15 36-57.

Rayner, K. (1998). Eye movements in reading and information processing: 20 years of research. Psychol. Bull. $124,372-422$

Rayner, K., and Well, A. D. (1996) Effects of contextual constraint on eye movements in reading: a further examination. Psychon. Bull. Rev. 3 504-509.

Sánchez-Casas, R. M., and GarcíaAlbea, J. E. (2005). "The representation of cognate and noncognate words in bilingual memory: can cognate status be characterized as a special kind of morphological relation?" in Handbook of Bilingualism: Psycholinguistic Approaches, eds J. F. Kroll and A. M. B. de Groot (New York: Oxford University Press), 226-250.

Schwanenflugel, P. J., and LaCount, K. L. (1988). Semantic relatedness and the scope of facilitation for upcoming words in sentences. J. Exp. Psychol. Learn. Mem. Cogn. 14, 344-354.

Schwartz, A. I., and Kroll, J. F. (2006). Bilingual lexical activation in sentence context. J. Mem. Lang. 55, 197-212.

Segui, J., and Grainger, J. (1990). Priming word recognition with orthographic neighbors: effects of relative prime-target frequency. J. Exp. Psychol. Hum. Percept. Perform. 16, 65-76.

Spivey, M. J., and Marian, V. (1999). Cross talk between native and second languages: partial activation of an irrelevant lexicon. Psychol. Sci. 10, 281-284.

Stanovich, K. E., and West, R. F. (1983). On priming by a sentence context. $J$. Exp. Psychol. Gen. 112, 1-36.

Titone, D., Libben, M., Mercier, J., Whitford, V., and Pivneva, I. (2011). Bilingual lexical access during L1 sentence reading: the effects of L2 knowledge, semantic constraint, and L1-L2 intermixing. J. Exp. Psychol. Learn. Mem. Cogn. 37, 1412-1431.

Van Assche, E., Drieghe, D., Duyck, W. Welvaert, M., and Hartsuiker, R. J. (2011). The influence of semantic constraints on bilingual word recognition during sentence reading. $J$. Mem. Lang. 64, 88-107.
Van Assche, E., Duyck, W., Hartsuiker, R. J., and Diependaele, K. (2009). Does bilingualism change nativelanguage reading? Cognate effects in a sentence context. Psychol. Sci. 20, 923-927.

van Hell, J. G., and de Groot, A. M. B. (1998). Conceptual representation in bilingual memory: effects of concreteness and cognate status in word association. Biling. (Camb. Engl.) 1 , 193-211.

van Hell, J. G., and de Groot, A. M. B. (2008). Sentence context modulates visual word recognition and translation in bilinguals. Acta Psychol. (Amst.) 128, 431-451.

van Hell, J. G., and Dijkstra, T. (2002). Foreign language knowledge can influence native language performance in exclusively native contexts. Psychon. Bull. Rev. 9, 780-789.

van Heuven, W. J. B., Dijkstra, T., and Grainger, J. (1998). Orthographic neighborhood effects in bilingual word recognition. J. Mem. Lang. 39, 458-483.

van Orden, G. C. (1987). A rows is a rose: spelling, sound, and reading. Mem. Cognit. 15, 181-198.

Weber, A., and Cutler, A. (2004). Lexical competition in non-native spokenword recognition. J. Mem. Lang. 50, $1-25$.

Conflict of Interest Statement: The authors declare that the research was conducted in the absence of any commercial or financial relationships that could be construed as a potential conflict of interest.

Received: 01 March 2012; accepted: 15 May 2012; published online: 01 June 2012.

Citation: Van Assche E, Duyck W and Hartsuiker RJ (2012) Bilingual word recognition in a sentence context. Front. Psychology 3:174. doi: 10.3389/fpsyg.2012.00174

This article was submitted to Frontiers in Language Sciences, a specialty of Frontiers in Psychology.

Copyright () 2012 Van Assche, Duyck and Hartsuiker. This is an open-access article distributed under the terms of the Creative Commons Attribution Non Commercial License, which permits noncommercial use, distribution, and reproduction in other forums, provided the original authors and source are credited. 